\title{
Catalytic activities of heterogeneous catalysts obtained by copolymerization of metal-containing 2-(acetoacetoxy)ethyl methacrylate
}

https://doi.org/10.1515/chem-2018-0055

received March 7, 2018; accepted March 18, 2018.

\begin{abstract}
Among the synthetic strategies commonly used for supporting a metal complex onto an organic polymer in order to obtain an heterogenous catalyst, a valid choice is to synthesize a metal containing monomer (MCM), which can subsequently be subjected to polymerization with suitable comonomers and crosslinkers, achieving a supported transition metal catalyst as a metal-containing polymer (MCP). In this context, during the last two decades, we explored the use of 2-(acetoacetoxy)ethyl methacrylate (HAAEMA) as a ligand to prepare several MCMs for the relevant MCPs. In this review we summarize and discuss our developments in the studies of the catalytic activity of these "hybrid" catalysts. These catalysts have demonstrated high efficiency and/or excellent selectivity in several kinds of chemical reactions and very often they could be recovered and reused in multiple cycles maintaining their activity and selectivity without suffering from appreciable metal leaching.
\end{abstract}

Keywords: supported transition metal catalysts; heterogeneous catalysts; metal-containing monomers; metal-containing polymers; reductions; oxidations; C-C bond forming reactions.

\section{Abbreviations}

AAEMA $^{-}$- deprotonated form of 2-(acetoacetoxy) ethyl methacrylate; AIBN - azobisisobutyronitrile (IUPAC name: 2,2'-azobis(2-methylpropionitrile)); BSA - N,O-bis(trimethylsilyl)acetamide ; cod - 1,5cyclooctadiene ; CP/MAS NMR - Cross Polarization/ Magic Angle Spinning Nuclear Magnetic Resonance; (+)-diop - (S,S)-4,5-bis(diphenylphosphinomethyl)-2,2'dimethyl-1,3-dioxolane; DCE - 1,2-dichloroethane; DEG - diethylene glycol; DMAA - N,N-dimethylacrylamide; DMF - N,N-dimethylformamide; EGDMA - ethylene glycol dimethacrylate; EMA - ethyl methacrylate; er enantiomeric ratio; MBAA-N,N'-methylenebisacrylamide; HAAEMA - 2-(acetoacetoxy)ethyl methacrylate (IUPAC name: 2-(2-methylprop-2-enoyloxy)ethyl 3-oxobutanoate); $(R, S)$-JOSIPHOS - $\quad(R)-1-\left[\left(S_{\mathrm{P}}\right)-2\right.$-(diphenylphosphino) ferrocenyl]ethyldicyclohexylphosphine; MCM - metalcontaining monomer; [ $(R, S)$-PPFA - $(R)$-N,N-dimethyl-1[(S)-2-(diphenylphosphino)ferrocenyl]ethylamine; TBAB - tetra-n-butyl ammonium bromide; TEM - transmission electron microscopy; THF - tetrahydrofuran; TOF turnover frequency;

\section{Introduction}

In the last three decades, a large number of researches have been dealt with the possibility of supporting a metal complex onto an insoluble matrix to obtain a heterogeneous catalyst. The matrixes on which metal complexes can be immobilized are generally inert inorganic materials (typically oxides) or organic polymers but the latter, by choosing a suitable combination of comonomers and crosslinkers, in principle allows to control the flexibility of the catalyst as well as to fine-tune its physical properties (polarity, swellability, morphology,

\footnotetext{
*Corresponding author: Giuseppe Romanazzi, DICATECh, Politecnico di Bari, Via E. Orabona 4, 70125 Bari, Italy, E-mail: giuseppe.romanazzi@poliba.it Piero Mastrorilli, Mario Latronico, Matilda Mali, Maria Michela Dell'Anna: DICATECh, Politecnico di Bari, Via E. Orabona 4, 70125 Bari, Italy

Angelo Nacci: Dipartimento di Chimica, Università di Bari "Aldo Moro", via E. Orabona 4, 70125 Bari, Italy
} 
etc.) [1-4]. The only disadvantages of this approach are sometimes the low thermal capacity and mechanical strength of the obtained heterogeneous catalysts [5]. The most commonly used strategies for synthesizing a supported metal complex on organic polymer or resin are essentially those represented in Scheme 1.

Route a is the "classic" synthesis of a macromolecular ligand followed by the linking of a transition metal as salt or complex. [2,6] Although the route $\mathbf{b}$ is less frequently used, it is more interesting both from a synthetic and a catalytic point of view. The route $\mathbf{b}$ consists of the preparation of a metal containing monomers (MCM), i.e. a catalytically active transition metal complex with a ligand having a functionality, which can subsequently be subjected to polymerization with suitable comonomers and crosslinkers for achieving a supported transition metal catalyst as a metal-containing polymer (MCP). Moreover, the use of MCMs offers the advantage of a possible comparison of the activity between the homogeneous and the heterogeneous phase as well as a more uniform distribution of the transition metal in the support with respect to route $\mathbf{a}$, and thus a more controllable dispersion of the metal in the polymer matrix. The use of MCMs for the obtainment of heterogeneous catalysts has been reviewed by some of us in the 2004 and more recently in 2013. [7,8] In this framework, during the last two decades, we explored the use of 2-(acetoacetoxy) ethyl methacrylate (HAAEMA) as a ligand to prepare several MCMs for the obtainment of relevant MCPs. HAAEMA (Scheme 2) is a clear or light-yellow liquid which finds use as versatile functional acrylic monomer [9-14] for making copolymers to be used in various applications such as, by way of example, dental resins, [15,16] coatings for glass and metal surfaces, [17] wound sealants, [18] waterborne coatings, [19,20] thermal nanoimprint lithography, [21] and nanoparticles [22-25]. On the other hand, exploiting the fact that the reactivity of the $\beta$-ketoester functionality in HAAEMA towards transition metal salts or complexes is very close to that of acetylacetone, we were able to prepare several transition metal complexes containing the ligand

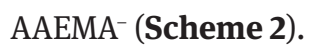

The spectroscopic features of all AAEMA- complexes demonstrate that the $\beta$-ketoester moiety is the sole functionality involved in the coordination while the methacrylic tail is indeed left free for other reactions, such as radical polymerizations. Thanks to these characteristics, it was possible to synthesise and employ in catalysis several polymer-supported transition metal catalysts obtained from copolymerization of transition metal-containing AAEMA- with appropriate comonomers and crosslinkers (Scheme 3). All resulting MCPs are nonhygroscopic powders which are insoluble in all solvents

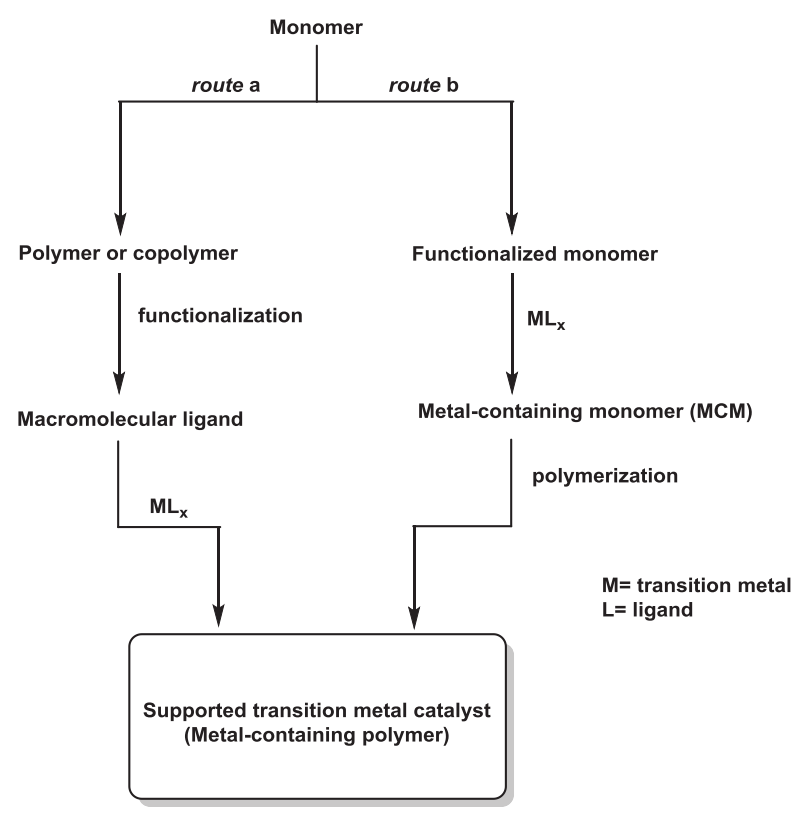

Scheme 1:
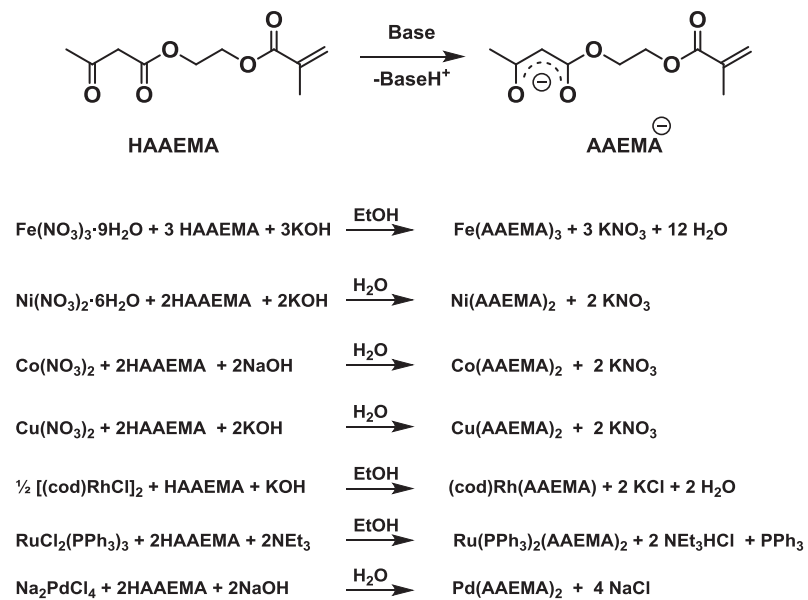

Scheme 2:

but they swelled well in water, acetone, halogenated solvents, dioxane, THF, DMF, and shrink when treated with diethyl ether, ethyl acetate or petroleum ethers. These swellabilities are very valuable in looking for new catalytic systems that could be recoverable and recyclable. In this review, we summarize and discuss our developments in the studies of catalytic activity of these "hybrid" catalysts. We will focus on their advantages in terms of recyclability without forgetting to point out some of their drawbacks. The discussion on the various MCPs has been subdivided into paragraphs each of which dealing with a single transition metal. 


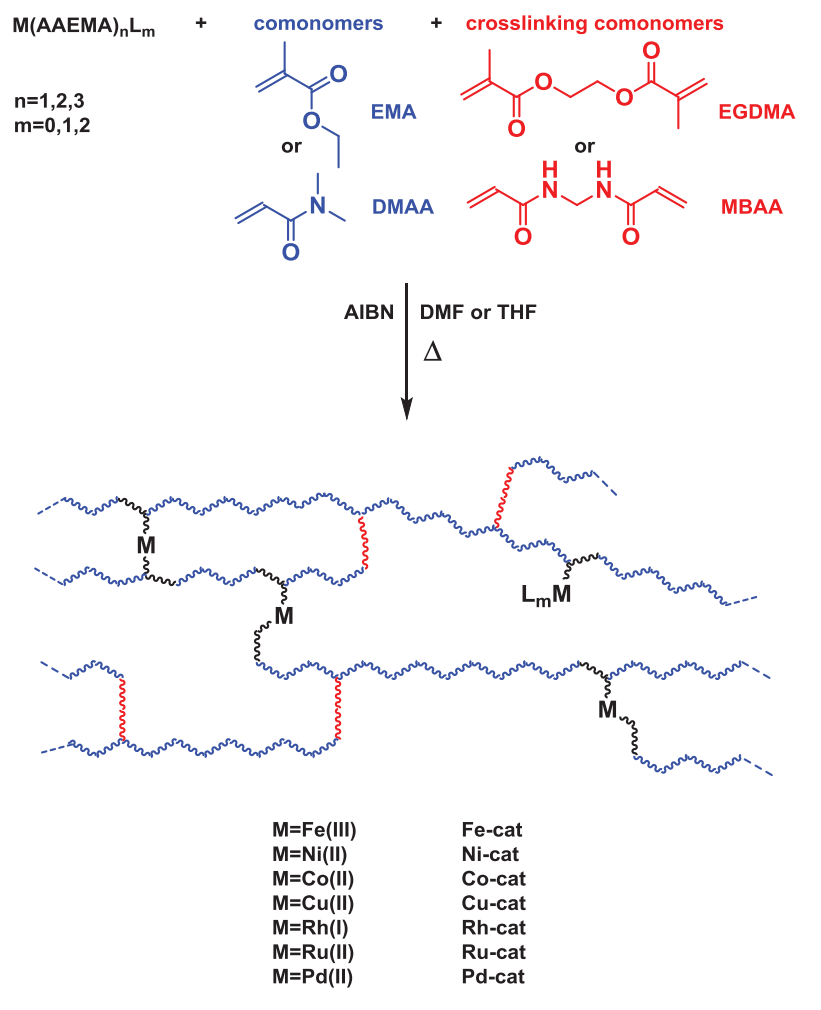

Scheme 3:

\section{Catalytic activity of Fe-cat}

Fe-cat was obtained as orange solid by copolymerizing the complex Fe(AAEMA) ${ }_{3}$ with DMAA as comonomer, and MBAA as crosslinker in DMF at $50^{\circ} \mathrm{C}$ for $24 \mathrm{~h}$ (Scheme 3) [26]. The first application of Fe-cat was in the catalytic aerobic epoxidation of olefins (Scheme 4) in presence of isovaleraldehyde as coreductant at room temperature in 1,2-dichloroethane (DCE). Under these reaction conditions, also known as "Mukaiyama's conditions", [27] Fe-cat proved to be highly active and selective for the epoxidation of unsaturated substrates such as norbornene, cholesteryl acetate, and cyclohexene. The catalytic system was recyclable without loss of activity except for the epoxidation of open chain alkenes such as 1-octene and 1-dodecene where Fe-cat suffered from a severe metal leaching.

Under "Mukaiyama's conditions", Fe-cat was also used as heterogeneous catalyst for the aerobic oxidation of $\alpha$-hydroxyketones to $\alpha$-diketones (Scheme 5) [ $\left.{ }^{28}\right]$.

With a very low amount of catalyst, substrates such as benzoin, 4,4'-dimethyl-benzoin, and anisoin were smoothly oxidized to corresponding $\alpha$-diketones in moderate to good yield, and Fe-cat was easily separable from the reaction mixture and recyclable at least once without suffering from metal leaching.

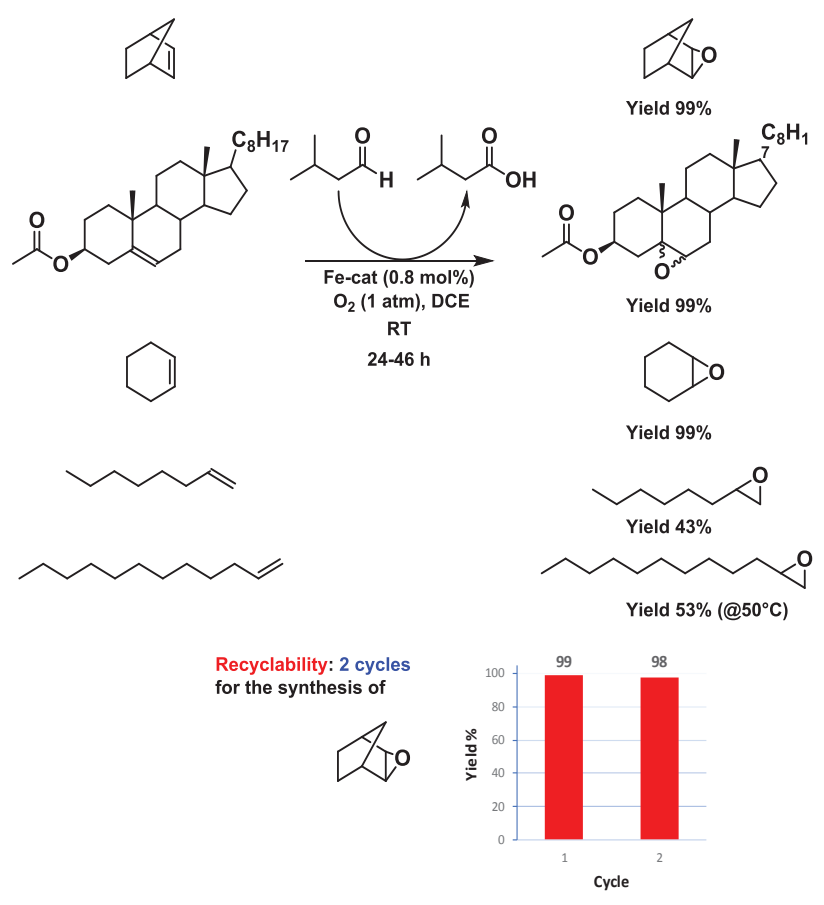

Scheme 4:

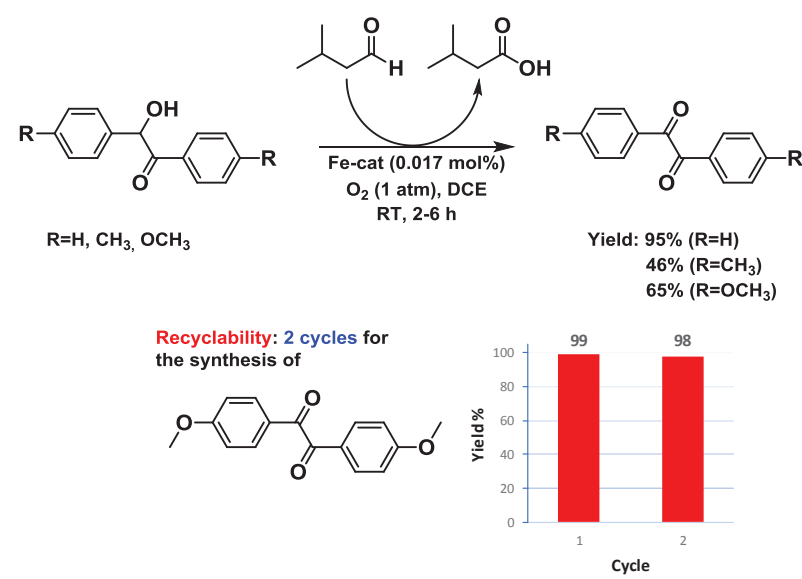

Scheme 5:

Finally, Fe-cat also catalysed a carbon-carbon bondforming reaction such as the cross-coupling between acyl chlorides and Grignard reagents, [29] affording several aliphatic or aromatic ketones in moderate to excellent yield (Scheme 6).

At least in one instance, it was possible to recycle Fe-cat, which showed a comparable activity and selectivity with respect to the first cycle. 


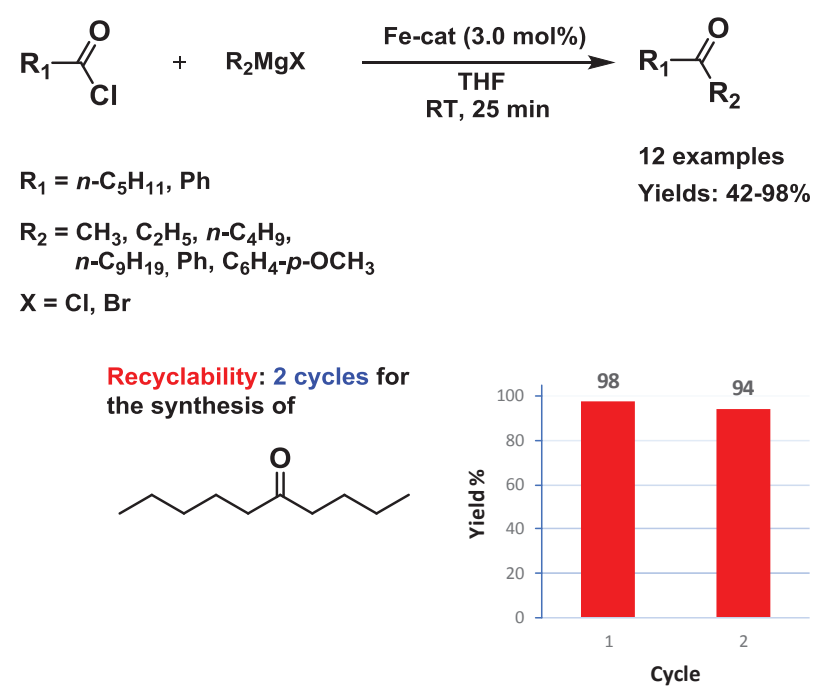

Scheme 6:

\section{Catalytic activity of Ni-cat}

Ni-cat was obtained as pale green powder by copolymerizing the complex Ni(AAEMA), with DMAA as comonomer, and MBAA as crosslinker in DMF at $120^{\circ} \mathrm{C}$ for $24 \mathrm{~h}$ (Scheme 3) [26].

The first application of Ni-cat in catalysis was the aerobic epoxidation of olefins under "Mukaiyama's conditions" and, like Fe-cat, Ni-cat proved to be active, selective, and recyclable for the epoxidation of norbornene, cholesteryl acetate, and cyclohexene. In the epoxidation of linear alkenes also this catalyst suffered from a severe metal leaching (Scheme 7).

The Ni-cat was also used as heterogeneous catalysts for Michael addition reactions (Scheme 8) [30].

When the Michael donor was methyl acetoacetate $\left(\mathrm{X}=\mathrm{CH}_{3} ; \mathrm{Y}=\mathrm{OCH}_{3}\right)$, the best yield $(92 \%)$ of the relevant adduct was achieved within $19 \mathrm{~h}$. The recycle test gave $76 \%$ yield after $24 \mathrm{~h}$. This case represents the best compromise in terms of activity and metal leaching (20\% of initial amount) of Ni-cat. For other donors, the adduct yields were lower and metal leaching for Ni-cat was even more significant (up to $87 \%$ of initial amount).

More recently, we explored the use of Ni-cat for accelerating the transfer hydrogenation of nitroarenes in aqueous media using $\mathrm{NaBH}_{4}$ as the reducing agent. Although preliminary catalytic tests using nitrobenzene as the representative substrate were encouraging (isolate yield up to 93\%), attempts to recycle Ni-cat failed. In the second run, the yield into aniline dropped to $27 \%$ due to severe metal leaching. Nevertheless, we found that by submitting Ni-cat to thermal annealing at $300^{\circ} \mathrm{C}$

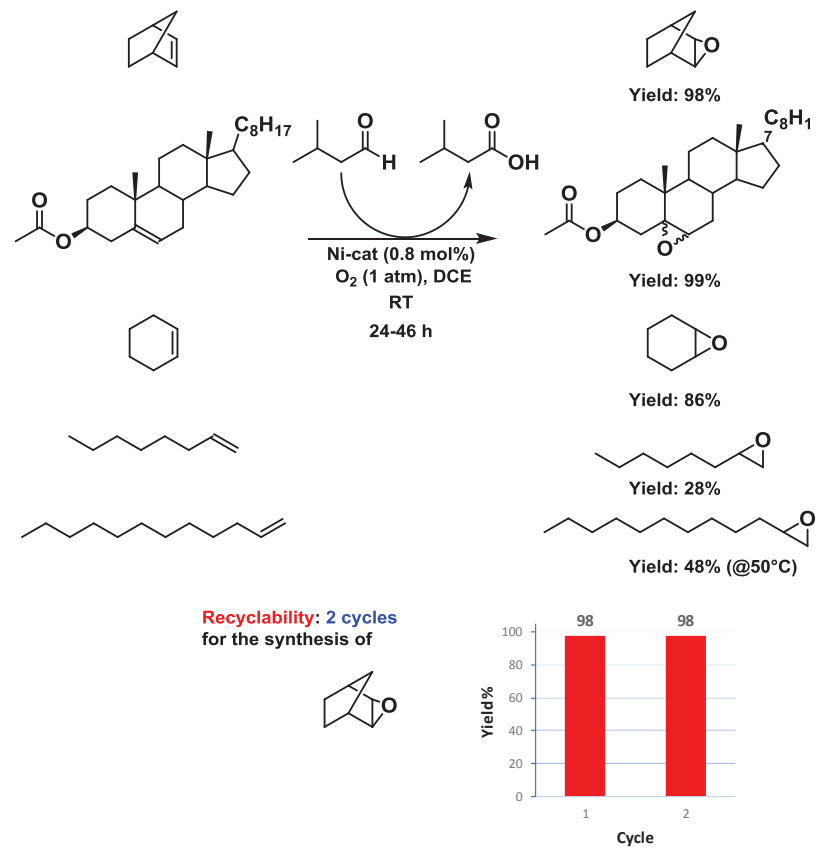

Scheme 7:
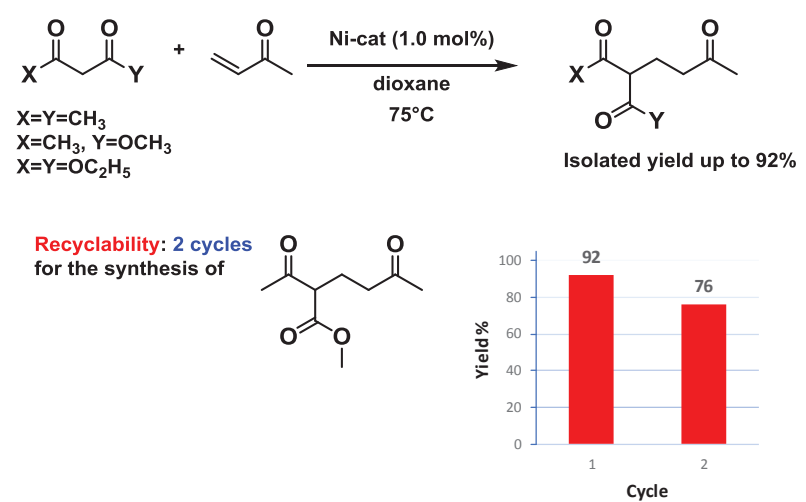

Scheme 8:

under nitrogen a polymer supported nickel nanomaterial (Ni-cat ${ }^{\#}$ ) was formed which proved to be a very active and selective catalyst for the hydrogenation of several functionalized nitroarenes to corresponding aromatic amines in aqueous medium at room temperature in the presence of $\mathrm{NaBH}_{4}$ (Scheme 9) [31].

Remarkably, Ni-cat ${ }^{\#}$ was found highly selective in the hydrogenation of halonitrobenzene to haloaniline, avoiding the formation of hydro-dehalogenated sideproducts, and displayed excellent recyclability for five cycles without suffering from metal leaching. 
<smiles>O=[N+]([O-])c1cccc(Cl)c1</smiles>

$$
\begin{gathered}
\underset{\mathrm{Ni}-\mathrm{Cat}^{\#}\left(1.86 \mathrm{~mol}^{2}\right)}{\longrightarrow} \\
\underset{20 \mathrm{eq} \mathrm{NaBH}_{4}}{\mathrm{NT}}
\end{gathered}
$$$$
\mathbf{G}=\mathrm{H}, \mathrm{CH}_{3}, \mathrm{OCH}_{3}
$$
$\mathrm{NO}_{2}, \mathrm{~F}, \mathrm{Cl}, \mathrm{Br}$,

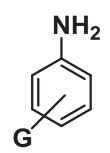

11 examples Yields: $\mathbf{8 2 - 9 9 \%}$
Recyclability: 5 cycles for the synthesis of<smiles>Nc1ccc(Br)cc1</smiles>

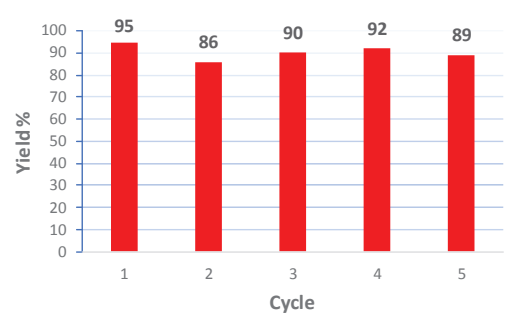

Scheme 9:

\section{Catalytic activity of Co-cat}

Co-cat was obtained as pink solid by copolymerizing the complex Co(AAEMA), with DMAA as comonomer, and MBAA as crosslinker in DMF at $50^{\circ} \mathrm{C}$ for $24 \mathrm{~h}$ (Scheme 3) [26]. In the aerobic epoxidation of olefins under “Mukaiyama's conditions", like Fe-cat and Ni-cat, Co-cat was active, selective, and recyclable for the epoxidation of norbornene, cholesteryl acetate, and cyclohexene but again a severe metal leaching was observed in the epoxidation of linear alkenes (Scheme 10).

Under "Mukaiyama's conditions", Co-cat acted as heterogeneous catalyst also for the aerobic oxidation of $\alpha$-hydroxyketones to $\alpha$-diketones, displaying a higher activity than Fe-cat (Scheme 11) [28].

Benzoin, 4,4'-dimethyl-benzoin, and anisoin were smoothly oxidized to the corresponding $\alpha$-diketones in good to excellent yields and Co-cat was recyclable at least once without appreciable metal leaching.

Moreover, Co-cat compared to Fe-cat and Ni-cat demonstrated to be the most versatile catalyst in the oxidation reactions. In fact, Co-cat was also an active and recyclable heterogeneous catalyst for the selective oxidation of disubstituted sulfides to sulfoxides and/or sulfones under "Mukaiyama's conditions" (Scheme 12 and Table 1) [32].

The selective formation of the intermediate sulfoxide was achieved by lowering the partial pressure of the oxidant, using dried air in place of pure dioxygen, and matching with the time reactions.

Having optimized the reaction conditions for the obtainment of sulfoxides, the highly regioselective oxidation of a bis-sulfide such as 2-(2-p-tolylsulfanyl-

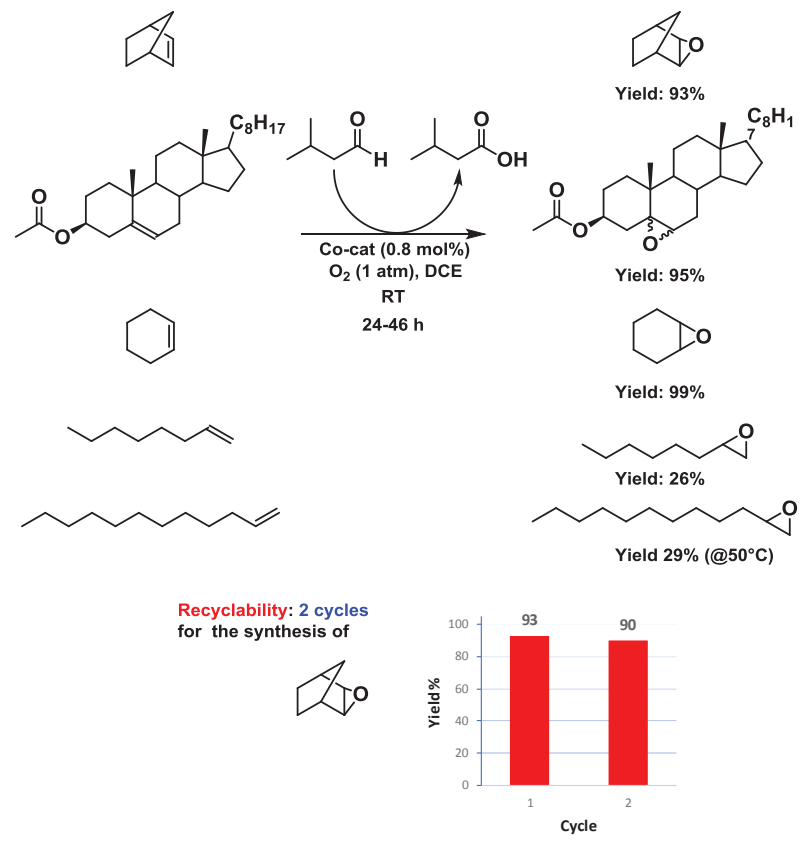

Scheme 10:
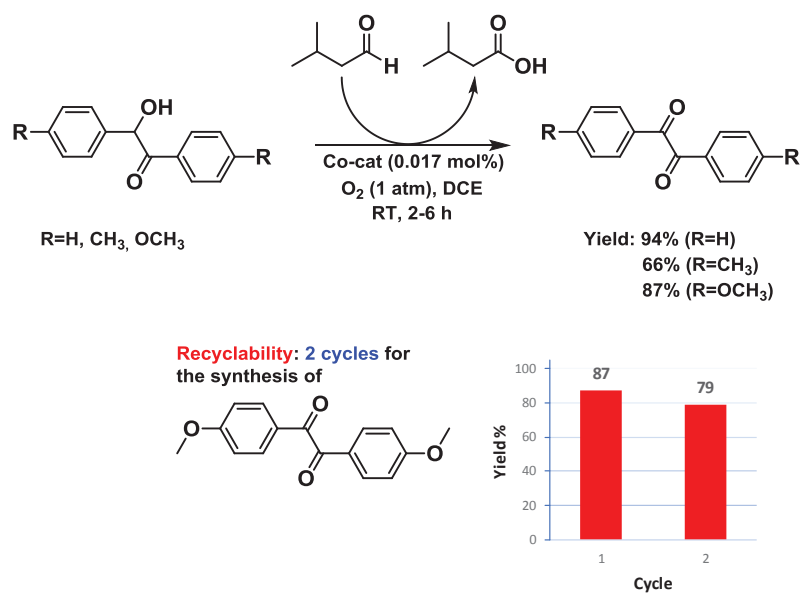

Scheme 11:

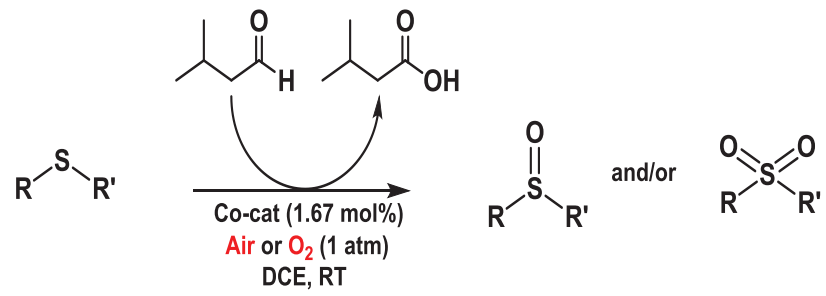

Scheme 12: 
Table 1: Oxidation of disubstituted sulphides catalysed by Co-Cat under Mukaiyama's conditions.

\begin{tabular}{llllll}
\hline Entry & Substrate & Oxidant & $\begin{array}{l}\text { Time } \\
\text { (h) }\end{array}$ & $\begin{array}{l}\text { Yield in } \\
\text { sulfoxide (\%) }\end{array}$ & $\begin{array}{l}\text { Yield in } \\
\text { sulfone (\%) }\end{array}$ \\
\hline 1 & $\left(\mathrm{CH}_{3}\right)_{2} \mathrm{~S}$ & Air & 8 & 80 & - \\
2 & $\left(\mathrm{CH}_{3}\right)_{2} \mathrm{~S}$ & Air & 20 & - & $>99$ \\
3 & $(n-\mathrm{Bu})_{2} \mathrm{~S}$ & Air & 21 & 83 & 10 \\
4 & $(n-\mathrm{Bu})_{2} \mathrm{~S}$ & Air & 37 & 8 & 92 \\
5 & $(t-\mathrm{Bu})_{2} \mathrm{~S}$ & $\mathrm{O}_{2}$ & 3 & - & $>99$ \\
6 & $(\mathrm{PhCH})_{2} \mathrm{~S}$ & $\mathrm{O}_{2}$ & 8 & - & $>99$ \\
7 & $p-\mathrm{Tol}_{-} \mathrm{S}-\mathrm{CH}_{3}$ & $\mathrm{O}_{2}$ & 2 & - & $>99$ \\
\hline
\end{tabular}

ethylsulfanyl)-benzothiazole into the corresponding sulfoxide 2-[2-(toluene-4-sulfinyl)-ethylsulfanyl]benzothiazole in $93 \%$ yield was successfully obtained (Scheme 13) [33].

Co-cat also acted as an active and reusable catalyst for the oxidation of benzylic and secondary alcohols at $40^{\circ} \mathrm{C}$ under "Mukaiyama's conditions" (Scheme 14) [34].

The corresponding carbonyl or ketone compounds were achieved in good to excellent yields and Co-cat was recycled at least four times without negligible metal leaching.

Pursuing our studies on the aerobic catalytic oxidation of organic substrates, we also found that Co-cat, under very mild conditions, behaved as a recyclable heterogeneous catalyst for the oxidation of alkyl and aryl phosphines to the corresponding oxides [35].

The Co-cat was an active heterogeneous catalyst for Michael addition reactions (Scheme 15) [30].

As in the case of Ni-cat, when the Michael donor was methyl acetoacetate $\left(\mathrm{X}=\mathrm{CH}_{3} ; \mathrm{Y}=\mathrm{OCH}_{3}\right)$, the best yield (97\%) of the relevant adduct was obtained within $19 \mathrm{~h}$ while a 93\% yield was obtained in the recycle test after 19h. Although Co-cat was a more active catalyst towards Michael reaction with respect to Ni-cat, its metal leaching was much higher. For example, in the case of methyl acetoacetate $\left(\mathrm{X}=\mathrm{CH}_{3} ; \mathrm{Y}=\mathrm{OCH}_{3}\right)$ it was $65 \%$ of initial amount (for Ni-cat, it was $20 \%$ of initial amount, see above).

\section{Catalytic activity of Cu-cat}

The supported copper complex Cu-cat, was obtained as green solid from copolymerization of $\mathrm{Cu}$ (AAEMA) with DMAA as comonomer, and MBAA as crosslinker in acetone/DMF (1/1) at $60^{\circ} \mathrm{C}$ for $5 \mathrm{~h}$ (Scheme 3). Cu-cat as Fe-cat and Co-cat was tested as metal catalyst in several
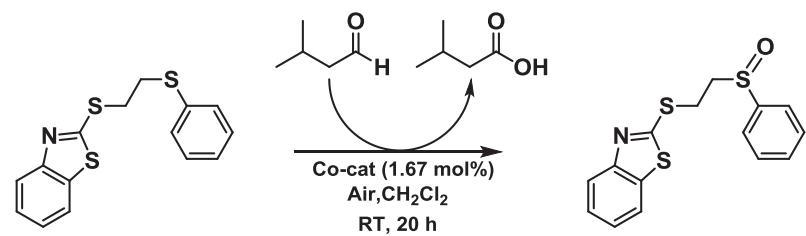

Yield: $93 \%$

Scheme 13:<smiles>OCc1ccccc1</smiles>
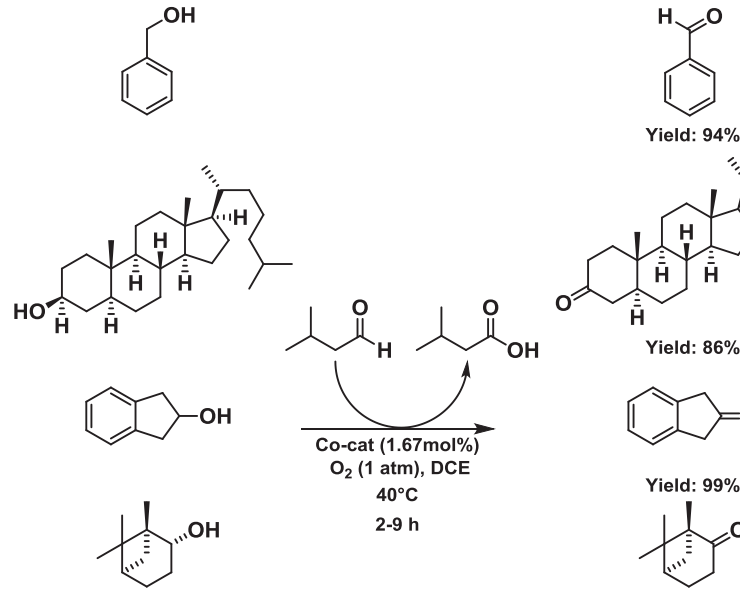

$2-9 \mathrm{~h}$
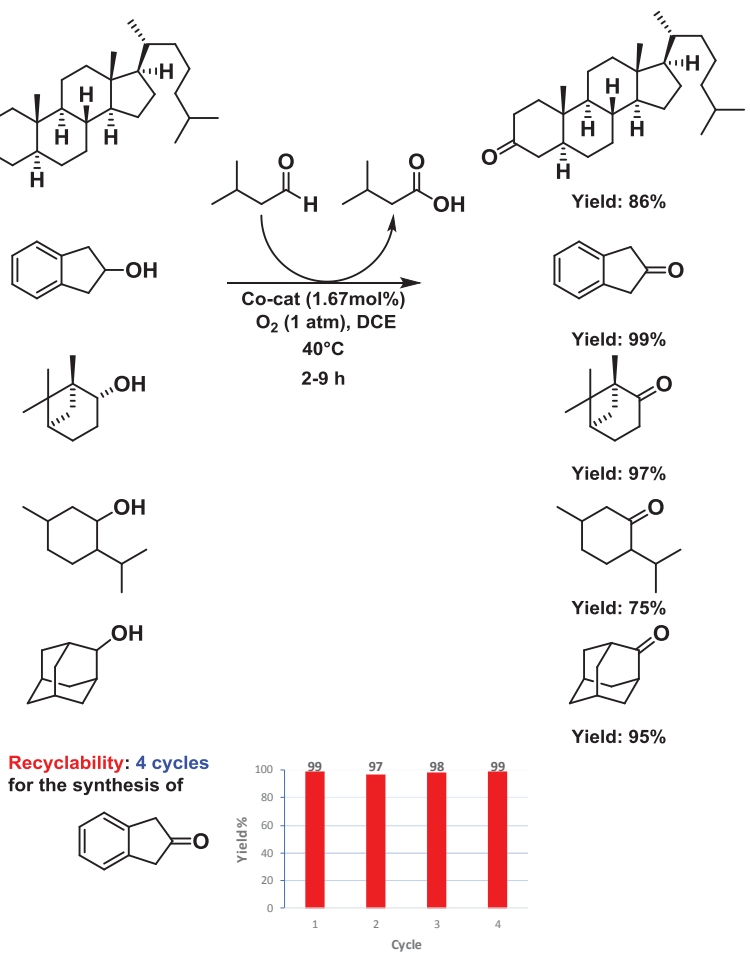

Yield: 95\%

Scheme 14:
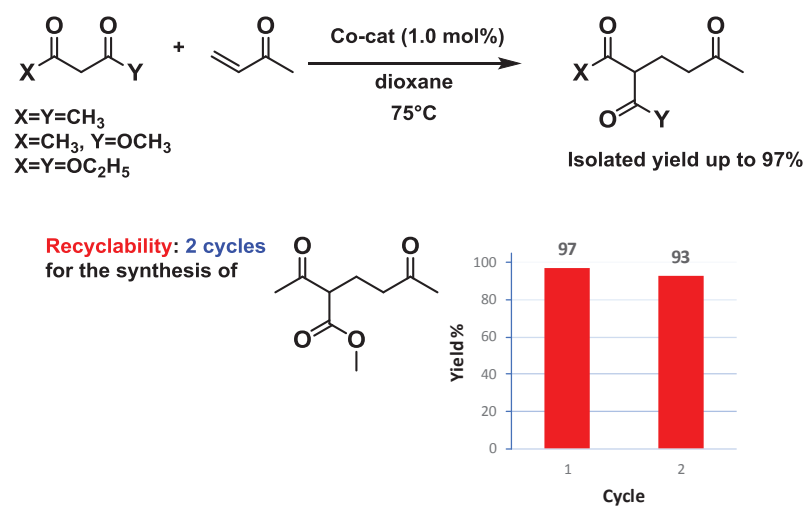

Scheme 15: 
Table 2: Hydrogenation of unsaturated substrates catalyzed by Rh-cat $(0.625 \% \mathrm{~mol})$.

\begin{tabular}{llllllll}
\hline Entry & Substrate & solvent & $\mathbf{T}\left({ }^{\circ} \mathrm{C}\right)$ & $\begin{array}{l}\mathbf{P} \\
\text { (bar) }\end{array}$ & Time (h) & Product & Yield (\%) \\
\hline 1 & 1-heptene & $\mathrm{CH}_{3} \mathrm{OH}$ & 21 & 1 & 4 & $n$-heptane & $>99$ \\
2 & cyclohexene & $\mathrm{CH}_{3} \mathrm{OH}$ & 21 & 1 & 2 & cyclohexane & $>99$ \\
3 & $(-)$-carvone & $\mathrm{CH}_{3} \mathrm{OH}$ & 21 & 1 & 6.5 & carvotanacetone & 77 \\
4 & citral & $\mathrm{CH}_{2} \mathrm{Cl}_{2}$ & 21 & 1 & 54 & citronellal & 64 \\
5 & cinnamaldehyde & $\mathrm{CH}_{2} \mathrm{Cl}_{2}$ & 21 & 20 & 8 & 3-phenylpropanal & 68 \\
6 & valeronitrile & $\mathrm{CH}_{3} \mathrm{OH}$ & 21 & 20 & 6 & dipentylamine & 85 \\
7 & nitrobenzene & $\mathrm{CH}_{3} \mathrm{OH}$ & 50 & 1 & 18 & aniline & $>99$ \\
\hline
\end{tabular}

oxidations under Mukaima's condition, but the obtained results were not encouraging with respect to them. On the other hand, Cu-cat was an active catalyst (Scheme 16) in the oxidative coupling of 2-naphthol for obtaining 1,1'-bi2-naphthol. However, the yield was moderate, and the catalyst was not recyclable [36].

\section{Catalytic activity of Rh-cat}

The synthesis of Rh-cat (Scheme 3) was achieved submitting the complex $\mathrm{Rh}(\mathrm{cod}) \mathrm{AAEMA}$ to copolymerization with DMAA and MBAA at $110^{\circ} \mathrm{C}$ in DMF in the presence of AIBN as radical initiator, obtaining an greenish-black powder. [37] Rh-cat catalysed the hydrogenation of several unsaturated substrates (Table 2) in methanol or $\mathrm{CH}_{2} \mathrm{Cl}_{2}$ with yields ranging from 64 to $>99 \%$ under very mild conditions.

Only cinnamaldehyde (entry 5), and valeronitrile (entry 6) required higher $\mathrm{H}_{2}$ pressure (20 bar) for their reduction. Five recycles of $\mathbf{R h}$-cat were made in the case of 1-heptene hydrogenation, without activity loss.

Encouraged by the good results achieved in nitrobenzene reduction (Table 2, entry 7), we employed $\mathbf{R h}$-cat in the reduction of $p$-halo-nitrobenzenes into $p$-halo-anilines (Scheme 17) [38].

Although the average turnover frequencies (TOF's) of catalysis were very low (from 0.95 up to $7.6 \mathrm{~h}^{-1}$ ), the selectivities were very high, thus minimizing concurrent hydrodehalogenation. In the recycle tests $\mathbf{R h}$-cat was even more active (average TOF's up to $12.0 \mathrm{~h}^{-1}$ ) but $6-8 \%$ of by-products such as p-halonitrosobenzene and 4,4'-dihaloazobenzene was also formed.

A chiral version (Rh-cat ${ }^{\star}$ ) of $\mathbf{R h}$-cat was synthesised by exchanging the cod ligand in (cod)Rh(AAEMA) with the $(S, S)$-DIOP and copolymerizing the resulting complex $[(+)$-diopRh(AAEMA)] with DMAA and MBAA in presence of AIBN in toluene/DMF at $100^{\circ} \mathrm{C}$ (Scheme 18) [39].
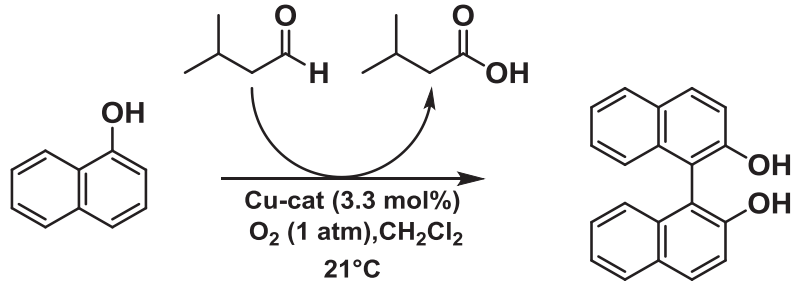

Yield 45\%

No Recyclability

Scheme 16:

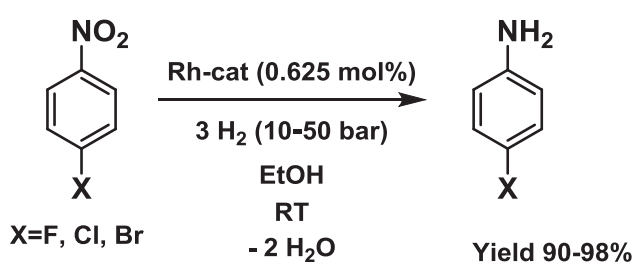

Recyclability: 2 cycles for the synthesis of<smiles>Nc1ccc(Cl)cc1</smiles>

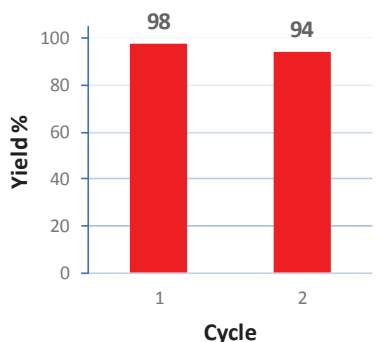

Scheme 17:

Rh-cat* accelerated the hydrogenation of methyl-(Z)$\alpha$-N-acetamidocinnamate to $\mathrm{N}$-acetyl-(S)-phenylalanine methyl ester with enantiomeric ratio (er) up to 76.5/23.5. However, Rh-cat ${ }^{\star}$ showed a lower enantioselectivity with respect to the homogeneous counterpart $[(S, S)$ DIOPRh(AAEMA)], which reached $e r=83.5 / 16.5$. Thanks to CP/MAS ${ }^{31} \mathrm{P}$ NMR studies [40-42], it was found that the lowering of enantioselectivity is due to the partial 
oxidation of the $(S, S)$-DIOP ligand that occurs during the copolymerization of $[(S, S)$-DIOPRh(AAEMA)]. In the recycle test reactions, $\mathbf{R h}^{-\mathbf{c a t}^{\star}}{ }^{\star}$ unfortunately halves its activity, lowering its enantioselectivity (er up to 65.0/35.0).

Finally, Rh-cat was also found to facilitate carboncarbon bond forming reactions. In fact, employing it as heterogeneous catalyst for the polymerization of phenylacetylene or $p$-tolylacetylene, polymers with excellent yields and very high stereoregularities were obtained [43]. In this framework, it was ascertained that $\mathbf{R h}$-cat could affect, in a reproducible manner, the size distribution of the nanospheres of the obtained polyphenylacetylenes [44].

\section{Catalytic activity of Ru-cat}

By copolymerizing the complex $\mathrm{Ru}\left(\mathrm{PPh}_{3}\right)_{2}(\mathrm{AAEMA})_{2}$ with DMAA as comonomer and MBAA in DMF and in presence of AIBN at $60^{\circ} \mathrm{C}$, the insoluble resin Ru-cat was obtained (Scheme 3) [45]. The catalytic figure of merit of Ru-cat were indeed modest: employing Ru-cat in the hydrogenation of alkenes in methanol at $65^{\circ} \mathrm{C}$ under 20 bar of $\mathrm{H}_{2}$, a low TOF $\left(16 \mathrm{~h}^{-1}\right)$ was achieved.

\section{Catalytic activity of Pd-cat}

Pd-cat is by far the most versatile and widely used catalyst among the family catalysts based on metalcontaining AAEMA because it was able to accelerate several kinds of chemical reaction such as reduction, oxidation and carbon-carbon bond forming reactions as well as esterification and transesterification reactions. Its synthesis requires the use of comonomer not containing nitrogen [37] and was achieved in very reproducible way by reacting $\operatorname{Pd}(\mathrm{AAEMA})_{2}$, ethyl methacrylate (EMA) and ethylene glycol dimethacrylate (EGDMA), this latter added in portions, in THF under the light of a table lamp in the presence of AIBN [46].

The first catalytic application of Pd-cat in a reduction reaction was the hydrogenation of several unsaturated substrates such as olefins, alkynes, unsaturated aldehydes, citral, carvone, and nitro compounds under $\mathrm{H}_{2}$ atmosphere at RT (Table 3) [47].

In most cases, the yields exceeded $90 \%$ within very short time reactions. Only benzaldehyde (entry 11 and 12) needed higher $\mathrm{H}_{2}$ pressure to be first transformed into benzyl alcohol $(\mathrm{t}=1 \mathrm{~h})$ and then into toluene $(4 \mathrm{~h})$.

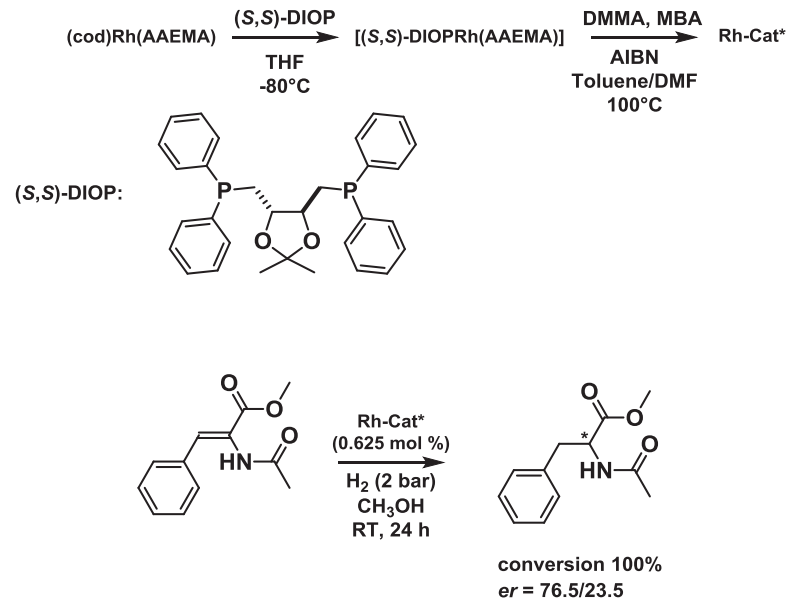

Scheme 18:

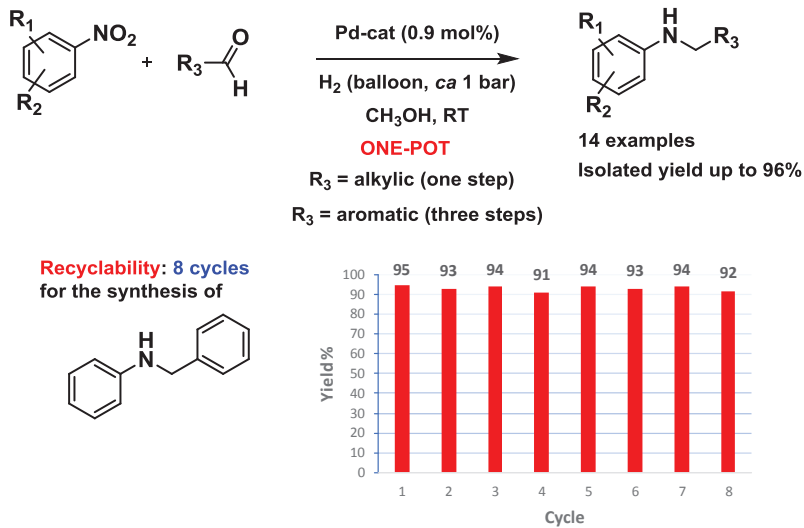

Scheme 19:

The refractoriness of benzaldehyde to be reduced prompted us to employ Pd-cat as a novel heterogenous catalyst in the one-pot direct amination reaction [48] using molecular hydrogen as reducing agent (Scheme 19) [49].

The catalytic system was found to be applicable, with slight differences, to both aliphatic and aromatic aldehydes and to a wide range of nitroarenes. Excellent yields of secondary amines were obtained under very mild conditions and the catalyst was recyclable at least 8 times without appreciable loss of activity and selectivity. TEM (transmission electron microscopy) analyses of Pd-cat demonstrated that the active species are insoluble palladium nanoparticles having a size distribution centred at $5 \mathrm{~nm}$.

In looking for an eco-friendly and economic catalytic system for the reduction of nitroarenes to arylamines, we also found that Pd-cat could catalyse such reduction in 
Table 3: Hydrogenation of unsaturated substrates catalyzed by Pd-cat $(0.625 \% \mathrm{~mol})$ at RT.

\begin{tabular}{lllllll}
\hline Entry & Substrate & solvent & $\mathbf{P}$ (bar) & Time (h) & Product & Yield (\%) \\
\hline 1 & 1-heptene & $\mathrm{CH}_{3} \mathrm{OH}$ & 1 & 2 & $n$-heptane & $>99$ \\
2 & cyclohexene & $\mathrm{CH}+\mathrm{OH}$ & 1 & 2 & cyclohexane & $>99$ \\
3 & 1-octyne & $\mathrm{CH}_{3} \mathrm{OH}$ & 1 & 5 & $n$-octane & 77 \\
4 & phenylacethylene & $\mathrm{CH}_{3} \mathrm{OH}$ & 1 & 7 & ethylbenzene & 99 \\
5 & diphenylacetylene & $\mathrm{CH}_{2} \mathrm{Cl}$ & 1 & 5.5 & 1,2-diphenylethane & $>99$ \\
6 & 2-cyclohexen-1-one & $\mathrm{CH}_{3} \mathrm{OH}$ & 1 & 2 & cyclohexanone & 91 \\
7 & 2-cyclopenten-1-one & $\mathrm{CH}_{3} \mathrm{OH}$ & 1 & 3 & cyclopentanone & $>99$ \\
8 & cinnamaldehyde & $\mathrm{CH}_{2} \mathrm{Cl}$ & 1 & 33 & 3-phenylpropanal & 83 \\
9 & citral & $\mathrm{CH}_{3} \mathrm{OH}$ & 1 & 10.5 & citronellal & 70 \\
10 & (-)-carvone & $\mathrm{CH}_{3} \mathrm{OH}$ & 1 & 7 & carvotanmenthone & 70 \\
11 & benzaldehyde & $\mathrm{CH}_{3} \mathrm{OH}$ & 10 & 1 & benzyl alcohol & 95 \\
13 & benzaldehyde & $\mathrm{CH}_{2} \mathrm{Cl}$ & 10 & 4 & toluene & $>99$ \\
15 & nitrobenzene & $\mathrm{CH}_{3} \mathrm{OH}$ & 1 & 7 & aniline & $>$ \\
\hline
\end{tabular}

the presence of $\mathrm{NaBH}_{4}$ and water as a mild reducing agent and a green solvent, respectively (Scheme 20) [50].

From moderate to excellent yields of aryl amines were obtained and the catalyst was recyclable at least 12 times without loss of activity and selectivity. In fact, TEM observations showed that the catalytic active species are very small Pd nanocrystallites (mean size diameter ca. 3 nm) formed in the presence of $\mathrm{NaBH}_{4}$.

The activity of Pd-cat in reduction reactions was also tested in the reduction of quinolines to 1,2,3,4-tetrahydroquinolines, which are important intermediates for the synthesis of biological active molecules, drugs, agrochemicals, dyes, and alkaloids [51]. However, the selective catalytic hydrogenation of polynuclear heteroaromatic nitrogen compounds such as quinolines is a difficult task because quinolines usually poison the traditional noble metal-based hydrogenation catalysts. [52-54] In spite of these assumptions, Pd-cat was found an active and selective catalyst for reduction of quinolines and quinoxalines (Scheme 21) [55].

In aqueous medium, high yields and excellent selectivity were achieved under not severe conditions $\left(80^{\circ} \mathrm{C}\right.$ and 10 bar of $\left.\mathrm{H}_{2}\right)$. The catalytic system maintained its activity and selectivity for nine cycles with negligible metal leaching. TEM analyses on catalyst pointed out that the active species were supported Pd nanoparticles, having a mean size of $4 \mathrm{~nm}$, which did not aggregate with the recycles.

Searching for an eco-friendly and more economic catalytic system for the reduction of quinolines to

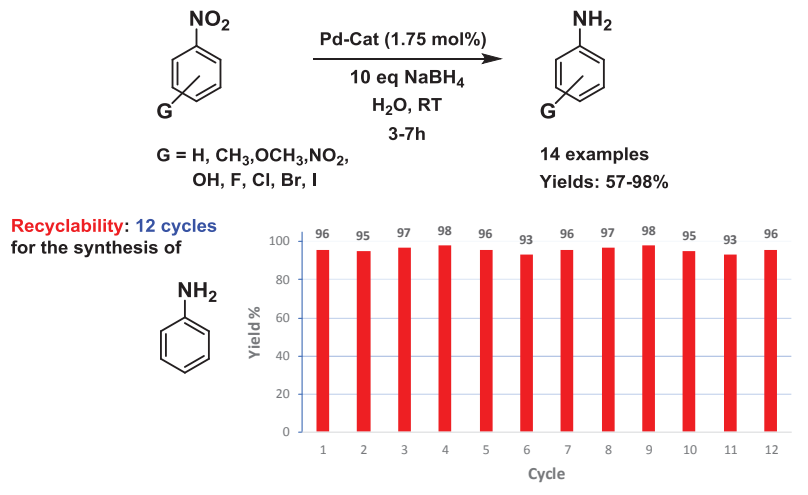

Scheme 20:

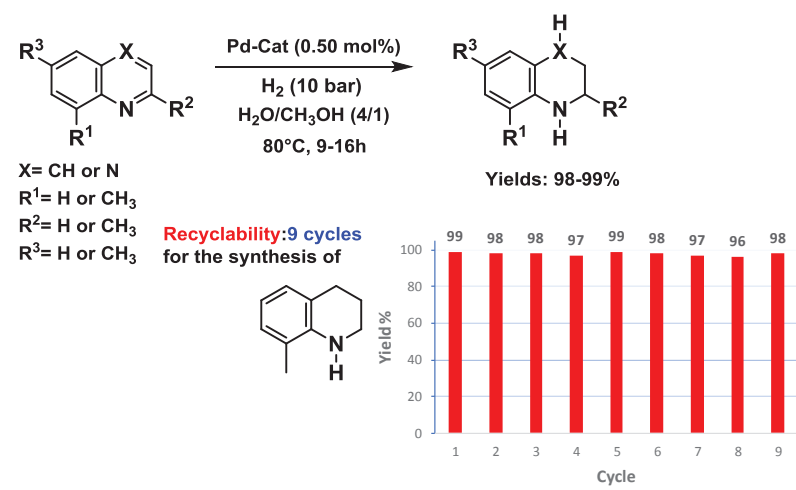

Scheme 21: 
1,2,3,4-tetrahydroquinolines under milder conditions, we found that Pd-cat catalysed the reduction of quinolines in the presence of $\mathrm{NaBH}_{4}$ as a mild reducing agent and neat water as green solvent (Scheme 22) [56].

Although a slightly higher Pd-cat loading (2.0 mol \%) was necessary, the catalytic system exhibited excellent activity and selectivity, which were maintained for at least seven reaction runs without metal leaching. By means of TEM studies, it was ascertained that the active species were supported Pd nanoparticles, having a smaller mean size (3 $\mathrm{nm}$ ) with respect to the protocol under $\mathrm{H}_{2}$ pressure.

The first application of Pd-cat in accelerating a carbon-carbon bond forming reaction was the Heck crosscoupling reaction [57-59] between aryl iodides or bromides and different olefins, in the presence of potassium or sodium acetate as base (Scheme 23), in which Pd-cat also acted as phosphane free catalyst [60].

A very low Pd-cat loading was necessary to achieve trans disubstituted-olefins in moderate to excellent yields and the catalyst was recycled up to six times with slight loss of its activity. However, when the reaction mixture was hot filtered at $20 \%$ conversion of aryl halide, the activity of the mother liquor was almost comparable to that of fresh Pd-cat. On the other hand, if the filtration was carried out at $100 \%$ conversion of aryl halide, the activity of mother liquors was experimentally negligible. Thus, Pd-cat behaved as a reservoir of catalytically active palladium soluble species, which deactivated when the aryl halide was consumed [61].

This behaviour was found to be not operating in the asymmetric allylic alkylation reaction [62-64] promoted by Pd-cat in the presence of catalytic amount of chiral ligands, such as $(R, S)$-PPFA, $(R, S)$-JOSIPHOS, and $(R, R)$ NORPHOS (Scheme 24) [65].

The reaction between rac-1,3-diphenyl-2-propenyl acetate with dimethyl malonate in the presence of N,Obis (trimethylsilyl) acetamide (BSA) and a catalytic amount of KOAc provided good yields and good enantioselectivity (er up to 96.5:3.5 in the presence of (R,S)-JOSIPHOS). However, only when chiral ferrocenyl ligands $(R, S)$-PPFA and $(R, S)$-JOSIPHOS) were employed, Pd-cat could be used in two subsequent runs with a severe metal leaching (for $(R, S$ )-JOSIPHOS: $71 \%$ yield and $e r=92.5 / 7.5$ in the recycle but metal leaching up to $39 \%$ of initial amount). Furthermore, in the case of $(R, R)$-NORPHOS the metal leaching after the first cycle is already almost quantitative.

Pd-cat also displayed high efficiency in the Stille cross-coupling [66,67] of aryl iodides or bromides with various trimethyltin or tributyltin derivatives (Scheme 25) [68].

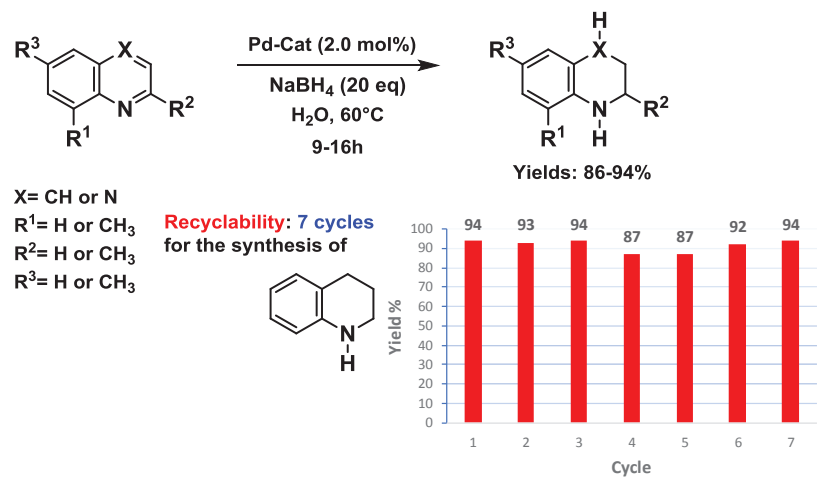

Scheme 22:

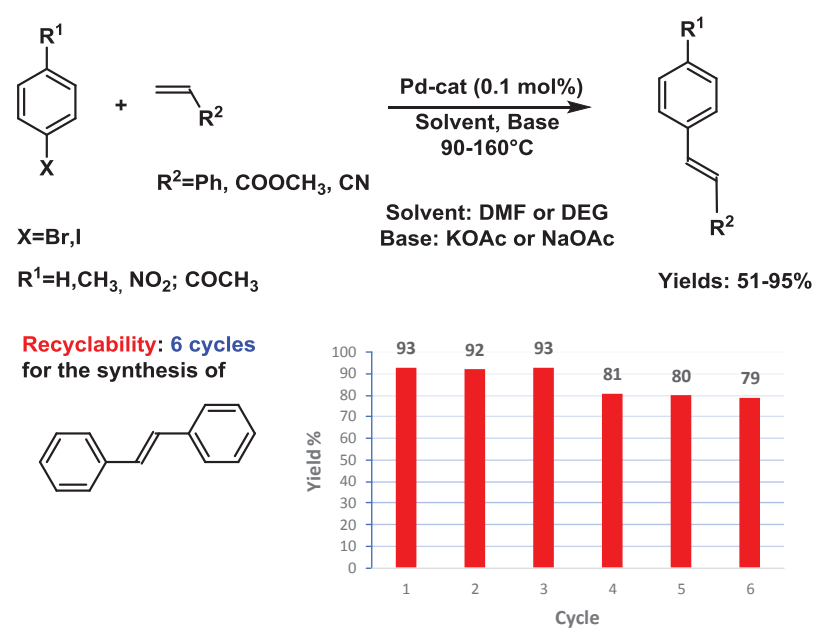

Scheme 23:
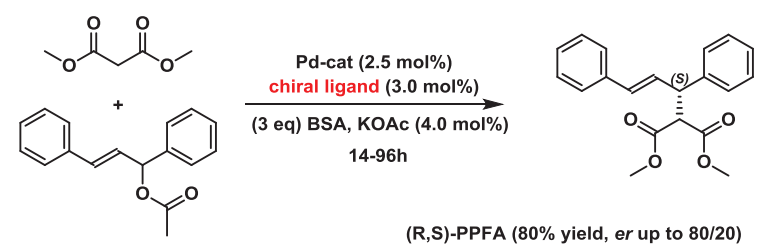

(R,S)-PPFA ( $80 \%$ yield, er up to $80 / 20$ ) (R,S)-JOSIPHOS (90\% yield, er up to $96.5: 3.5$ ) (R,R)-NORPHOS (60\% yield, er up to $84.5: 15.5$ )

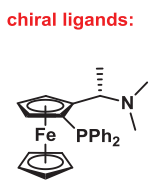

$(R, S)$-PPFA

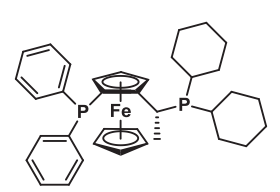

$(R, S)$-JOSIPHOS

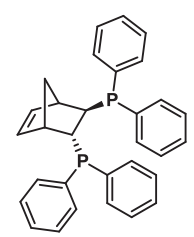

$(R, R)$-NORPHOS
Scheme 24: 
Yields from 63 to 99\% were achieved in first cycle. In the case of aryl iodides, the catalyst was recycled up to six runs with appreciable lowering of catalytic activity due to the accumulation of tin derivatives onto the support, which hampered the migration of the substrates to the catalytically active sites.

Pd-cat was also successfully used as catalyst in the Suzuki-Miyaura cross-coupling reaction [69-72] of aryl bromides (or activated aryl chlorides) under air in neat water at $100^{\circ} \mathrm{C}$, in the presence of $\mathrm{K}_{2} \mathrm{CO}_{3}$ as base (Scheme 26) [73].

From good to excellent yields in biaryls were achieved and the catalyst was recycled at least five times without suffering from a significant palladium leaching. By means of hot filtration tests of the mother liquors, we established that Pd-cat acted as a reservoir of catalytically active species (palladium nanoparticles) that leached out in a very little amount for each cycle in solution. The use of a transfer phase agent, such as tetrabutylammonium bromide (TBAB) accelerated the cross-coupling reaction because it facilitated the formation of smaller size (more active) palladium nanoparticles.

The good activity and reusability of Pd-Cat in water media for the reduction of nitroarenes or quinolines with $\mathrm{H}_{2}$ or $\mathrm{NaBH}_{4}$ as reducing agent, and for the Suzuki-Miyaura cross-coupling reaction pushed us to perform a detailed and accurate studies on palladium nanoparticles formed during their catalytic runs. By combining information obtained through high-resolution TEM, X-ray energy dispersive spectroscopy, and micro-IR spectroscopy we were able to study the morphological features of the Pd nanoparticles formed in Pd-cat, before and after its use in catalysis, and also the effect on their evolution in function of several parameters, such as different reducing agents (aryl boronic acid, dihydrogen, $\mathrm{NaBH}_{4}$ ), the presence of a phase transfer (TBAB) and the temperature [74]. Micro-IR spectroscopy showed that the support was chemically stable over the catalyst recycles in all the reactions tested, while TEM analyses pointed out that the macroporosity of the resin remained constant after each run and in all cases the pristine $\mathrm{Pd}(\mathrm{II})$ polymer supported complex was reduced in situ to $\mathrm{Pd}(0)$ forming nanoparticles (the active species) under reaction conditions. The organic support was always able to stabilize Pd nanoparticles during recycling without negligible agglomeration. High temperatures $\left(80-100^{\circ} \mathrm{C}\right)$ favoured the formation of Pd nanoparticles of $9 \mathrm{~nm}$ average size, while at room temperature the $\mathrm{Pd}$ nanoparticles average size was smaller (2-6 nm).

The presence of a phase transfer agent (TBAB) led to formation of $\mathrm{Pd}$ nanoparticles with $4 \mathrm{~nm}$ average size,

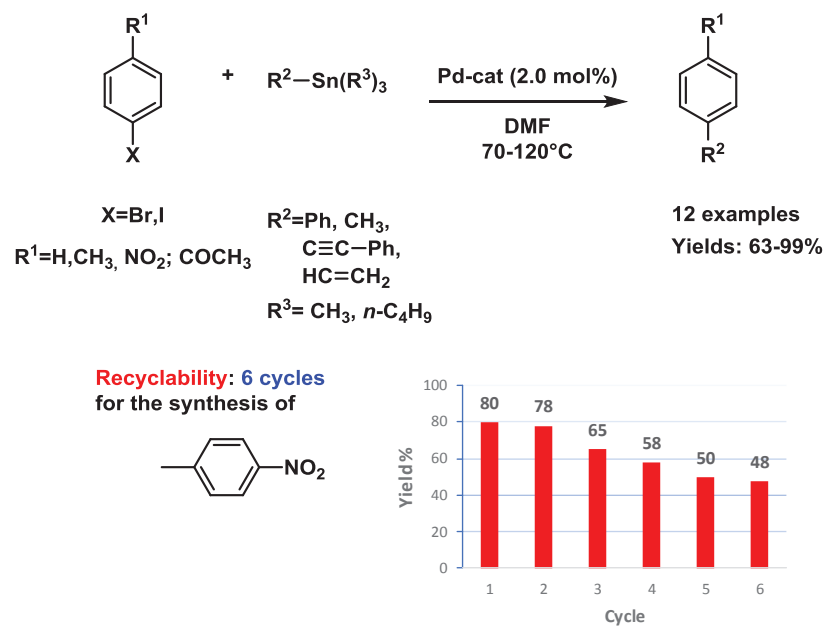

Scheme 25:
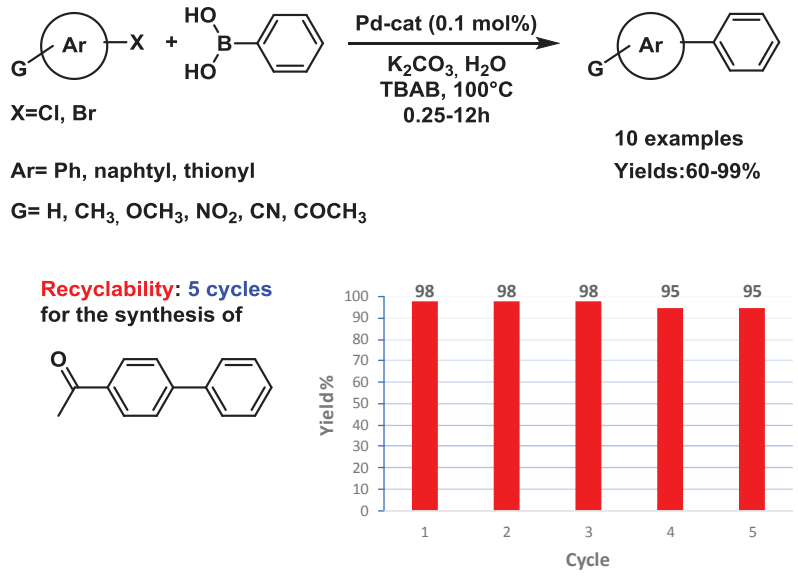

Scheme 26:

which leached into solution because of the well-known capacity of TBAB to stabilize the nanoparticles favouring their solubilization [75-77].

Concerning the use of different reducing agents on Pd nanoparticles, in the Suzuki-Miyaura coupling, the effect of the reductant such as aryl boronic acid was masked by the temperature reaction $\left(100^{\circ} \mathrm{C}\right)$ and/or by the presence of TBAB (when used). On the contrary, for the reduction of nitroarenes or quinolines in water, the choice of the reductant $\left(\mathrm{H}_{2}\right.$ or $\left.\mathrm{NaBH}_{4}\right)$ was fundamental to determinate the diameter size of Pd nanoparticles. Under $\mathrm{H}_{2}$ atmosphere, Pd nanoparticles with average diameter size from 6 to $10 \mathrm{~nm}$ were obtained, while in the presence of $\mathrm{NaBH}_{4}$ they ranged from 2 to $4 \mathrm{~nm}$.

By summarizing the investigation results, in SuzukiMiyaura reaction Pd-cat operateed as a reservoir of 
soluble Pd nanoparticles, which were released into the aqueous environment and they were effectively recaptured by the polymer matrix after substrate consumption. In fact, TEM images of Pd-cat recovered after any recycles showed the majority of Pd nanoparticles onto the surface of the polymer. This "release and catch" mechanism of active species, is commonly accepted by the scientific community for some heterogeneously catalysed $\mathrm{C}-\mathrm{C}$ bond forming reaction [78-80]. On the other hand, in the hydrogenation reactions under $\mathrm{H}_{2}$ atmosphere or in the presence of $\mathrm{NaBH}_{4}$, Pd-cat operated by means of a truly heterogeneous catalytic mechanism because TEM images of Pd-cat recovered after any recycles showed a uniform distribution of Pd nanoparticles in the polymer matrix.

Pd-cat acted as heterogeneous catalyst for the oxidation of alcohols in water by air. Primary and secondary aromatic alcohols were oxidized to their corresponding carbonyl compounds in excellent yields with a very low Pd loading $(0.5 \mathrm{~mol} \%)$ in relatively short reaction times (Scheme 27) [81].

The catalyst was easily recovered and reused up to six cycles. Hot filtration tests indicated that Pd-cat really operated through a heterogeneous pathway.

Finally, Pd-cat was found to catalyse, under pressure of 2 bar of $\mathrm{H}_{2}$ and in the presence of catalytic amount of bromobenzene in excess of alcohols, the esterification (or transesterification) of aliphatic and aromatic carboxylic acids into their corresponding esters in good to excellent yields (Scheme 28) [82].

Bromobenzene was necessary to generate in-situ a little amount of $\mathrm{HBr}$, which provided a mild acidic reaction environment. Under these reaction conditions the true active species were, again, stabilized Pd nanoparticles and Pd-cat was reusable for eight consecutive cycles with a negligible Pd leaching into solution. The catalytic system was also explored in preliminary studies for the preparation of partially hydrogenated fatty acid methyl esters, starting from a mixture composed by highly polyunsaturated esters and free carboxylic acids, taken as a model acidic feedstock for mimic a biodiesel upgrading $[83,84]$.

\section{Conclusions}

Polymerization of transition metal-containing the polymerizable ligand AAEMA turned out to be a convenient alternative tool for the preparation of supported transition metal catalysts as MCPs. These MCPs employed as catalysts in several kinds of chemical reactions showed<smiles>[R]c1ccc(C([R])O)cc1</smiles>

$\mathbf{R}^{1}=\mathrm{H}$ or alky $\mathrm{R}^{2}=\mathrm{H}, \mathrm{F}, \mathrm{NO}_{2}, \mathrm{OCH}_{3}$

Recyclability: 6 cycles for the synthesis of<smiles>O=Cc1ccccc1</smiles>

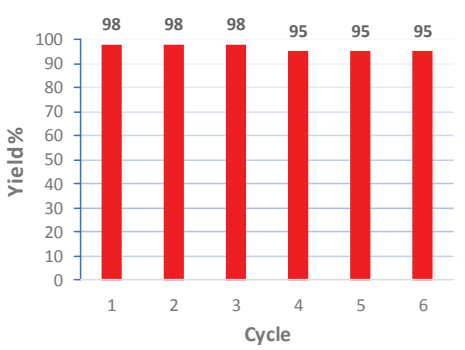

Scheme 27:

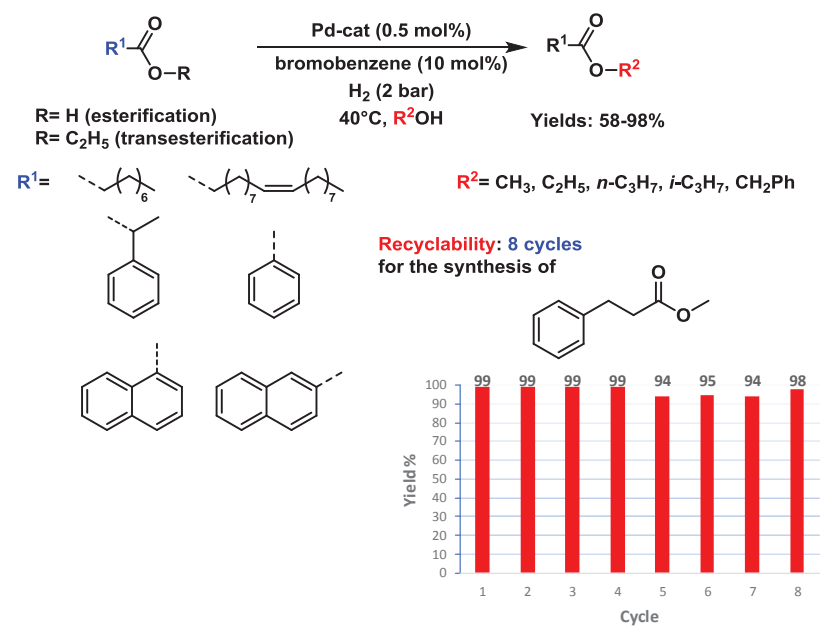

Scheme 28:

high efficiency and/or excellent selectivity and very often they could be recovered and reused in multiple cycles without significant loss of their activity and selectivity. Soon, other catalytic applications will be explored for these catalysts and new noble or non-noble transition metal-containing AAEMA will be synthesized and then polymerized with suitable comonomers and crosslinkers to obtain novel catalysts. Much efforts will be made to systematically ascertain when the solid matrix not only possess the role of heterogenizing the catalyst but also when it affects the chemical efficiency and the selectivity of a chemical process. 
Acknowledgements: G.R., P.M., M.L. and M.M.D. acknowledge Politecnico di Bari for Fondi di Ricerca di Ateneo (FRA2016).

Conflict of interest: Authors state no conflict of interest.

\section{References}

[1] Lu J., Toy P.H., Organic Polymer Supports for Synthesis and for Reagent and Catalyst Immobilization, Chem. Rev., 2009, 109, 815-838.

[2] Dioos B.M.L., Vankekecom I.F.J., Jacobs P.A., Aspects of Immobilisation of Catalysts on Polymeric Supports, Adv. Synth. Catal. 2006, 348, 1413-1446.

[3] Leadbeater N.E., Marco M., Preparation of Polymer-Supported Ligands and Metal Complexes for Use in Catalysis, Chem. Rev., 2002, 102, 3217-3274.

[4] Hîbner S., de Vries J.G., Farina V., Why Does Industry Not Use Immobilized Transition Metal Complexes as Catalysts? Adv. Synth. Catal. 2016, 358, 3-25.

[5] Hartley F. R. (1987) Supported metal complex catalysts, in The Metal - Carbon Bond: Volume 4 (1987) (ed Hartley F. R.), John Wiley \& Sons, Inc., Chichester, UK.

[6] Kaliyappan T., Kannan P., Co-ordination polymers, Prog. Polym. Sci., 2000, 25, 343-370.

[7] Mastrorilli P., Nobile C.F., Supported catalysts from polymerizable transition metal complexes, Coord. Chem. Rev., 2004, 248, 377-395.

[8] Dell'Anna M.M., Romanazzi G., Mastrorilli P., Polymer Supported Catalysts Obtained from Metal-Containing Monomers, Curr. Org. Chem., 2013, 17, 1236-1273.

[9] Zoller A., Kockler K.B., Rollet M., Lefay C., Gigmes D., BarnerKowollik C., Guillaneuf Y., A complete kinetic study of a versatile functional monomer: acetoacetoxyethyl methacrylate (AAEMA), Polym. Chem., 2016, 7, 5518-5525.

[10] Schlaad H., Krasia T., Antonietti M., Superhelices of Poly[2(acetoacetoxy)ethyl methacrylate], J. Am. Chem. Soc., 2004, 126, 11307-11310.

[11] Hiltl S., Schürings M.-P., Balaceanu A., Mayorga V., Liedel C., Pich A., Böker A., Guided self-assembly of microgels: from particle arrays to anisotropic nanostructures, Soft Matter, 2011,7, 8231-8238.

[12] Krasia T., Soula R., Börner H.G., Schlaad H., Controlled synthesis of homopolymers and block copolymers based on 2-(acetoacetoxy)ethyl methacrylate via RAFT radical polymerization, Chem. Commun., 2003, 538-539.

[13] Levy A., Gaver E., Wang F., Galant O., Diesendruck C.E., The effect of intramolecular cross links on the mechanochemical fragmentation of polymers in solution, Chem. Commun., 2017, 10132-10135.

[14] Pal S., Das A., Maiti S., De P., Synthesis and characterization of a biodegradable polymer prepared via radical copolymerization of 2-(acetoacetoxy)ethyl methacrylate and molecular oxygen, Polym. Chem., 2012, 3, 182-189.

[15] Viljanen E.K., Skrifvars M., Vallittu P.K., Dendritic copolymers and particulate filler composites for dental applications:
Degree of conversion and thermal properties, Dent. Mater., 2007, 23, 1420-1427.

[16] Viljanen E.K., Langer S., Skrifvars M., Vallittu P.K., Analysis of residual monomers in dendritic methacrylate copolymers and composites by HPLC and headspace-GC/MS, Dent. Mater., 2006, 22, 845-851.

[17] Pospiech D., Jehnichen D., Starke S., Müller F., Bünker T., Wollenberg A., Häußler L., Simon F., Grundke K., Oertel U., Opitz M., Kruspe R., Multifunctional methacrylate-based coatings for glass and metal surfaces, Appl. Surf. Sci., 2017, 399, 205-214.

[18] Wu J.J., Shek P.N., Synthesis and characterization of a novel in situ forming gel based on hydrogel dispersions, J. Biomed. Mater. Res. B Appl. Biomater., 2009, 90B, 738-44.

[19] González I., Arzamendi G., Asua J.M., Leiza J.R., Unexpected Crosslinking During Acetoacetoxy Group Protection on Waterborne Crosslinkable Latexes, Macromol. Mater. Eng., 2006, 291, 1185-1193.

[20] González I., Asua J.M., Leiza J.R., Crosslinking in Acetoacetoxy Functional Waterborne Crosslinkable Latexes, Macromol. Symp. 2006, 243, 53-62.

[21] Dinachali S.S., Saifullah M.S.M., Ganesan R., Thian E.S., He C., A Universal Scheme for Patterning of Oxides via Thermal Nanoimprint Lithography, Adv. Funct. Mater., 2013, 23, 2201-2211.

[22] Zhou W., Qu Q.,Yu W., An Z., Single Monomer for Multiple Tasks: Polymerization Induced Self-Assembly, Functionalization and Cross-Linking, and Nanoparticle Loading, ACS Macro Lett., 2014, 3, 1220-1224.

[23] Papaphilippou P., Loizou L., Popa N.C., Han A., Vekas L., Odysseos A., Krasia-Christoforou T., Superparamagnetic Hybrid Micelles, Based on Iron Oxide Nanoparticles and Well-Defined Diblock Copolymers Possessing $\beta$-Ketoester Functionalities, Biomacromolecules, 2009, 10, 2662-2671.

[24] Papaphilippou P., Christodoulou M., Marinica O.-M., Taculescu A., Vekas L., Chrissafis K., Krasia-Christoforou T., Multiresponsive Polymer Conetworks Capable of Responding to Changes in pH, Temperature, and Magnetic Field: Synthesis, Characterization, and Evaluation of Their Ability for Controlled Uptake and Release of Solutes, ACS Appl. Mater. Interfaces 2012, 4, 2139-2147.

[25] Sanchez-Sanchez A., Pomposo J., Efficient Synthesis of Single-Chain Polymer Nanoparticles via Amide Formation, J. Nanomater., 2015, 2015, 1-7.

[26] Dell'Anna M.M., Mastrorilli P., Nobile C.F., Suranna G.P., Epoxidation of olefins catalysed by polymer-supported metal $\beta$-ketoesterato complexes of iron (III), nickel (II) and cobalt (II), J. Mol. Catal. A-Chem., 1995, 103, 17-22.

[27] Mukaiyama T. (1993) Oxygenation of Olefins with Molecular Oxygen Catalyzed by Low Valent Metal Complexes. In: Barton D.H.R., Martell A.E., Sawyer D.T. (eds) The Activation of Dioxygen and Homogeneous Catalytic Oxidation. Springer, Boston, MA.

[28] Dell'Anna M.M., Mastrorilli P., Nobile C.F., Lopez L., Aerobic oxidation of $\alpha$-hydroxyketones catalysed by cobalt(II) and iron(III) complexes under homogeneous and heterogeneous conditions, J. Mol. Catal. A-Chem., 1996, 111, 33-36.

[29] Dell'Anna M.M., Mastrorilli P., Nobile C.F., Marchese G., Taurino M.R., Coupling reactions between acyl chlorides and Grignard 
reagents in the presence of a supported Fe(III) -complex, J. Mol. Catal. A-Chem., 2000, 161, 239-243.

[30] Mastrorilli P., Nobile C.F., Suranna G.P., Catalytic activity of polymerizable metal $\beta$-ketoesterato complexes towards Michael addition reactions, J. Mol. Catal. A-Chem., 1995, 103, 23-29.

[31] Romanazzi G., Fiore A. M., Mali M., Rizzuti A., Leonelli C., Nacci A., Mastrorilli P., Dell’Anna M.M., Polymer supported Nickel nanoparticles as recyclable catalyst for the reduction of nitroarenes to anilines in aqueous medium, Mol. Catal., 2018, 446, 31-38.

[32] Dell'Anna M.M., Mastrorilli P., Nobile C.F., Aerobic oxidation of sulfides catalysed by cobalt(II) complexes under homogeneous and heterogeneous conditions, J. Mol. Catal. A-Chem., 1996, 108, 87-62.

[33] Dell'Anna M.M., Mastrorilli P., Nobile C.F., Taurino M.R., Calò V., Nacci A., Regioselective aerobic oxidation of bis-sulfides into monosulfoxides, J. Mol. Catal. A-Chem., 2000, 151, 61-69.

[34] Cicco S.R., Latronico, M., Mastrorilli P., Suranna G.P., Nobile C.F., Homogeneous and heterogeneous catalytic oxidation of benzylic and secondary alcohols with a metal dioxygenato complex in the presence of 2-methylpropanal and dioxygen, J. Mol. Catal. A-Chem., 2001, 165, 135-140.

[35] Mastrorilli P., Muscio F., Nobile C.F., Suranna G.P., Aerobic oxidation of trivalent phosphorus and arsenic compounds in the presence of 3-methylbutanal and metal catalyst, J. Mol. Catal. A-Chem., 1999, 148, 17-21.

[36] Mastrorilli P., Muscio F., Suranna G.P., Nobile C.F., Latronico M., Aerobic oxidation of substituted phenols catalysed by metal acetylacetonates in the presence of 3-methylbutanal, J. Mol. Catal. A-Chem., 2001, 165, 81-87.

[37] Dell'Anna M.M., Mastrorilli P., Rizzuti A., Suranna G.P., Nobile C.F., Synthesis and copolymerization of rhodium(I) and palladium(II) complexes with the deprotonated form of 2-(acetoacetoxy)ethyl methacrylate, Inorg. Chim. Acta, 2000, 304, 21-25.

[38] Dell’Anna M.M., Mastrorilli P., Gallo V., Romanazzi G., A Recyclable Nanoparticle-Supported Rhodium Catalyst for Hydrogenation Reactions, Molecules, 2010, 15, 3311-3318.

[39] Mastrorilli P., Rizzuti A., Romanazzi G., Suranna G.P., Gobetto R., Nobile C.F., Synthesis and catalytic activity of new supported rhodium(I) complexes for the enantioselective hydrogenation of methyl-(Z)- $\alpha-\mathrm{N}$-acetamidocinnamate, J. Mol. Catal. A-Chem., 2002, 180, 177-185.

[40] Clark H.C., Davies J.A., Fyfe C.A., Hayes P.J., Wasylishen R.E., Investigations of polymer-supported complexes of platinum(II) by high-resolution solid-state phosphorus-31 NMR spectroscopy employing magic-angle spinning and crosspolarization techniques, Organometallics, 1983, 2, 177-180.

[41] Fyfe C.A., Davies J.A., Clark H.C., Hayes P.J., Wasylishen R.E., Polymer-immobilized complexes of platinum(II): their precursors and preparation studied by high-resolution solid-state phosphorus-31 NMR using magic-angle spinning techniques, J. Am. Chem. Soc., 1983, 105, 6577-6584.

[42] Gutmann T., Grünberg A., Rothermel N., Werner M., Srour M., Abdulhussain S., Tan S., Xu Y., Breitzke H., Buntkowsky G., Solid-state NMR concepts for the investigation of supported transition metal catalysts and nanoparticles, Solid State Nucl. Magn. Reson., 2013, 55-56, 1-11.
[43] Mastrorilli P., Nobile C.F., Rizzuti A., Suranna G.P., Acierno D., Amendola E., Polymerization of phenylacetylene and of $\mathrm{p}$-tolylacetylene catalyzed by $\beta$-dioxygenato rhodium(I) complexes in homogeneous and heterogeneous phase, J. Mol. Catal. A-Chem., 2002, 178, 35-42.

[44] Mastrorilli P., Nobile C.F., Suranna G.P., Corradi A., Leonelli C., Veronesi P., Morphological characterization of poly(phenylacetylene) nanospheres prepared by homogeneous and heterogeneous catalysis, Appl. Organometal. Chem., 2003, $17,711-716$.

[45] Mastrorilli P., Nobile C.F., Suranna G. P., Taurino M. R., Latronico M., Synthesis and copolymerization of a ruthenium(II) complex with the deprotonated form of 2-(acetoacetoxy) ethylmetacrylate, Inorg. Chim. Acta, 2000, 335, 107-112.

[46] Dell'Anna M.M., Mastrorilli P., Nobile C.F., Solid-Phase Catalytic Activity of a polymer-supported palladium complex. In SolidPhase Organic Syntheses; Scott P.J.H., Ed.; John Wiley \& Sons, Inc.: Hoboken, NJ, USA, 2012, pp. 79-86.

[47] Dell'Anna M.M., Gagliardi M., Mastrorilli P., Suranna G.P., Nobile C.F., Hydrogenation reactions catalysed by a supported palladium complex, J. Mol. Catal. A-Chem., 2000, 158, 515-520.

[48] Tripathi R.P., Verma S.S., Pandey J., Tiwari V.K., Recent Development on Catalytic Reductive Amination and Applications, Curr. Org. Chem., 2008, 12, 1093-1115.

[49] Dell’Anna M.M., Mastrorilli P., Rizzuti A., Leonelli C., One-pot synthesis of aniline derivatives from nitroarenes under mild conditions promoted by a recyclable polymer-supported palladium catalyst, Appl. Catal. A, 2011, 401, 134-140.

[50] Dell’Anna, M.M., Intini S., Romanazzi G., Rizzuti A., Leonelli C., Piccinni F., Mastrorilli, P., Polymer supported palladium nanocrystals as efficient and recyclable catalyst for the reduction of nitroarenes to anilines under mild conditions in water, J. Mol. Catal. A-Chem., 2014, 395, 307-314.

[51] Sridharan V., Suryavanshi P., Menendez J.C., Advances in the Chemistry of Tetrahydroquinolines, Chem. Rev., 2011, 111, 7157-7259.

[52] Zhou Y.-G., Asymmetric Hydrogenation of Heteroaromatic Compounds, Acc. Chem. Res., 2007, 40, 1357-1366.

[53] Dobereiner G.E., Nova A., Schley N.D., Hazari N., Miller S.J., Eisenstein O., Crabtree R.H., Iridium-Catalyzed Hydrogenation of N-Heterocyclic Compounds under Mild Conditions by an Outer-Sphere Pathway, J. Am. Chem. Soc., 2011, 133, 75477562, and references therein.

[54] Fish R.H., Tan J.L., Thormodsen A.D., Homogeneous catalytic hydrogenation. 4. Regioselective reduction of polynuclear heteroaromatic compounds catalyzed by hydridochlorotris(triphenylphosphine)ruthenium(II), Organometallics, 1985, 4 ,1743-1747.

[55] Dell'Anna M.M., Capodiferro V.F., Mali M., Manno D., Cotugno P., Monopoli A., Mastrorilli, P., Highly selective hydrogenation of quinolines promoted by recyclable polymer supported palladium nanoparticles under mild conditions in aqueous medium, Appl. Catal. A, 2014, 481, 89-95.

[56] Dell'Anna M.M., Romanazzi G., Intini, S., Rizzuti A., Leonelli C., Piccinni A.F., Mastrorilli P., A polymer supported palladium(II) $\beta$-ketoesterate complex as active and recyclable pre-catalyst for selective reduction of quinolines in water with sodium borohydride, J. Mol. Catal. A-Chem., 2015, 402, 83-91. 
[57] Beletskaya I.P., Cheprakov A.V., The Heck Reaction as a Sharpening Stone of Palladium, Catalysis, Chem. Rev., 2000, 100, 3009-3066.

[58] Jagtap S., Heck Reaction-State of the Art, Catalysts, 2017, 7, 267.

[59] For Heck reactions carried out in aqueous media, see: Christoffel F., Ward T.R., Palladium-Catalyzed Heck CrossCoupling Reactions in Water: A Comprehensive Review, Catal. Lett., 2018, 148, 489-511.

[60] Dell'Anna, M.M., Mastrorilli, P., Muscio, F., Nobile, C.F., Suranna G.P., A polymer-supported $\beta$-ketoesterate complex of palladium as an efficient, phosphane-free, air-stable, recyclable catalyst for the Heck reaction, Eur. J. Inorg. Chem., 2002, 1094-1099.

[61] Dell'Anna, M.M., Mastrorilli, P., Muscio, F., Nobile, C.F., A New Polymer Supported Palladium Complex as Active, Air Stable and Recyclable Catalyst for Carbon-Carbon Bond Forming Reactions, Stud. Surf. Sci. Catal., 2003, 145, 133-136.

[62] Trost B.M., VanVranken, D.L., Asymmetric transition metalcatalyzed allylic alkylations, Chem. Rev., 1996, 96, 395-422.

[63] Trost B.M., Crawley M.L., Asymmetric transition-metalcatalyzed allylic alkylations: Applications in total synthesis, Chem. Rev., 2003, 103, 2921-2943.

[64] Lu Z., Ma S., Metal-Catalyzed Enantioselective Allylation in Asymmetric Synthesis, Angew. Chem. Int. Ed., 2008, 47, 258-297.

[65] Dell'Anna M.M., Mastrorilli P., Nobile C.F., Suranna G.P., Asymmetric allylic alkylation using a polymer-supported palladium catalyst in the presence of chiral ligands, J. Mol. Catal. A-Chem., 2003, 201, 131-135.

[66] Farina V., Krishnamurthy V., Scott W. K., The Stille Reaction; Wiley: New York, 1998.

[67] Cordovilla C., Bartolomé C., Martínez-Ilarduya J.M., Espinet P., The Stille Reaction, 38 Years Later, ACS Catal., 2015, 5, 3040-3053.

[68] Dell'Anna M.M., Lofù A., Mastrorilli P., Mucciante V., Nobile C. F., Stille coupling reactions catalysed by a polymer supported palladium complex, J. Organomet. Chem., 2006, 691, 131-137.

[69] Miyaura N., Suzuki A., Palladium-Catalyzed Cross-Coupling Reactions of Organoboron Compounds, Chem. Rev., 1995, 96, 2457-2483.

[70] Suzuki A., Cross-Coupling Reactions Of Organoboranes: An Easy Way To Construct C-C Bonds (Nobel Lecture), Angew. Chem. Int. Ed., 2011, 50, 6723-6737.

[71] For Suzuki-Miyaura reactions catalyzed by metal nanoparticles, see: Fihri A., Bouhrara M., Nekoueishahraki B., Basset J.-M., Polshettiwar V., Nanocatalysts for Suzuki cross-coupling reactions, Chem. Soc. Rev., 2011, 40, 5181-5203.

[72] For Suzuki-Miyaura reactions carried out in aqueous media, see: Chatterjee A., Ward T.R., Recent Advances in the Palladium Catalyzed Suzuki-Miyaura Cross-Coupling Reaction in Water, Catal. Lett., 2016, 146, 820-840.
[73] Dell’Anna M.M., Mali M., Mastrorilli P., Rizzuti A., Ponzoni C., Leonelli C., Suzuki-Miyaura coupling under air in water promoted by polymer supported palladium nanoparticles, J. Mol. Catal. A-Chem., 2013, 366, 186-194.

[74] Mastrorilli P., Dell'Anna M.M., Rizzuti A., Mali M., Zapparoli M., Leonelli C., Resin-Immobilized Palladium Nanoparticle Catalysts for Organic Reactions in Aqueous Media: Morphological Aspects, Molecules, 2015, 20, 18661-18684.

[75] Reetz M.T., Westermann E., Phosphane-free palladiumcatalyzed coupling reactions: The decisive Role of Pd nanoparticles, Angew. Chem. Int. Ed., 2000, 39, 165-168.

[76] You E., Li P., Wang L., Nickel(0)/Tetra-n-butylammonium Bromide (TBAB) Catalyzed Suzuki-Miyaura Reaction, Synthesis, 2006, 1465-1469.

[77] Kumar S., Rao G.K., Kumar A., Singh M.P., Singh A.K., Palladium(II)-(E,N,E) pincer ligand $(\mathrm{E}=\mathrm{S} / \mathrm{Se} / \mathrm{Te})$ complex catalyzed Suzuki coupling reactions in water via in situ generated palladium quantum dots, Dalton Trans., 2013, 42, 16939-16948.

[78] Gruttadauria M., Giacalone F., Noto R., "Release and catch" catalytic systems, Green Chem., 2013, 15, 2608-2618.

[79] Ohtaka A., Okagaki T., Hamasaka G., Uozumi Y., Shinagawa T.; Shimomura O., Nomura R., Application of "boomerang" linear polystyrene-stabilized Pd nanoparticles to a series of C-C coupling reactions in water, Catalysts, 2015, 5, 106-118.

[80] Eremin D.B., Ananikov V.P., Understanding active species in catalytic transformations: From molecular catalysis to nanoparticles, leaching, "Cocktails" of catalysts and dynamic systems, Coord. Chem. Rev., 2017, 346, 2-19.

[81] Dell'Anna M.M., Mali M., Mastrorilli P., Cotugno P., Monopoli A., Oxidation of benzyl alcohols to aldehydes and ketones under air in water using a polymer supported palladium catalyst, J. Mol. Catal. A-Chem., 2014, 386, 114-119.

[82] Dell'Anna M.M., Capodiferro V.F., Mali M., Mastrorilli P., Esterification, transesterification and hydrogenation reactions of polyunsaturated compounds catalyzed by a recyclable polymer supported palladium catalyst, J. Organomet. Chem., 2016, 818,106-114.

[83] Guo M.X., Song W.P., Buhain J., Bioenergy and biofuels: History, status, and perspective, Renew. Sust. Energ. Rev., 2015, 42, 712-725.

[84] Chew T. L., Bhatia S., Catalytic processes towards the production of biofuels in a palm oil and oil palm biomassbased biorefinery, Bioresour. Technol., 2008, 99, 7911-7922. 